\title{
A systematic review of factors influencing NHS health check uptake: invitation methods, patient characteristics, and the impact of interventions
}

\author{
Amanda Bunten ${ }^{1 *}$ (D) Lucy Porter ${ }^{1,2}$, Natalie Gold ${ }^{1}$ and Vanessa Bogle ${ }^{3}$
}

\begin{abstract}
Background: The NHS Health Check (NHSHC) is a risk assessment for those aged 40-74 without a pre-existing condition in England, with the aim of preventing stroke, kidney disease, heart disease, type 2 diabetes and dementia. Uptake has been lower than anticipated. Ensuring that a high percentage of eligible patients receive a $\mathrm{NHSHC}$ is key to optimising the clinical and cost effectiveness of the programme. The aim of this systematic review is to highlight interventions and invitation methods that increase the uptake of NHSHCs, and to identify whether the effectiveness of these interact with broader patient and contextual factors.

Method: A systematic review was conducted according to the PRISMA checklist. Papers were eligible if they explored the impact of at least one of (i) interventions, (ii) invitation methods or (iii) broader factors on NHSHC uptake. Ten databases were searched in January 2016 and seven were searched in March 2018. Nine-hundred-and-forty-five papers were identified, 238 were screened and 64 full texts were assessed for eligibility. Nine studies were included in the review.

Results: The nine studies were all from peer reviewed journals. They included two randomised controlled trials, one observational cohort and six cross-sectional studies. Different invitation methods may be more effective for different groups of patients based on their ethnicity and gender. One intervention to enhance invitation letters effectively increased uptake but another did not. In addition, individual patient characteristics (such as age, gender, ethnicity and risk level) were found to influence uptake. This review also finds that uptake varies significantly by GP practice, which could be due either to unidentified practice-level factors or deprivation.

Conclusions: Further research is needed to assess the effectiveness of different invitation methods for different population groups. Research should examine how existing invitation methods can be enhanced to drive uptake whilst reducing health inequalities.
\end{abstract}

Trial registration: This systematic review was registered with PROSPERO on 22.02.2016. Registration number CRD42 016035626.

Keywords: NHS health check, Cardiovascular disease, Primary prevention, Uptake, Attendance, General practice, Invitation

\footnotetext{
* Correspondence: Amanda.bunten@phe.gov.uk

${ }^{1}$ Public Health England, PHE Behavioural Insights Team (PHEBI), Research,

Translation \& Innovation Division, 6th Floor, Wellington House, 133-155

Waterloo Road, London SE1 8UG, UK

Full list of author information is available at the end of the article

(c) Crown copyright will apply to Amanda Bunten, Lucy Porter and Natalie Gold. 2020 Open Access This article is distributed under the terms of the Creative Commons Attribution 4.0 International License (http://creativecommons.org/licenses/by/4.0/) which permits unrestricted use, distribution, and reproduction in any medium, provided you give appropriate credit to the original author(s) and the source, provide a link to the Creative Commons license, and indicate if changes were made. The Creative Commons Public Domain Dedication waiver (http://creativecommons.org/publicdomain/zero/1.0/) applies to the data made available in this article, unless otherwise stated.
} 


\section{Background}

Cardiovascular disease (CVD) is the number one cause of death globally; an estimated 17.9 million people died from CVD in 2016, representing 31\% of all global deaths [1]. It is estimated that 50 to $80 \%$ of CVD cases are caused by modifiable risk factors such as smoking, obesity, high blood pressure, high cholesterol, excessive alcohol consumption and physical inactivity, suggesting that the majority of cases are preventable [2].

In England, the National Health Service Health Check (NHSHC) provides a unique opportunity to target many CVD risk factors [2]. Introduced in 2009 by the Department of Health [3], the programme involves inviting everyone aged 40 to 74 years (who has not previously been diagnosed with CVD) to attend a NHSHC every 5 years, where their risk of heart disease, stroke, kidney disease and diabetes is assessed. The aims of this population prevention programme are (i) to reduce the risk of CVD in the population and subsequently the incidence of CVD events by providing individuals with support to make behavioural changes that will prevent the development of CVD, (ii) to facilitate early diagnosis of conditions such as high blood pressure, high cholesterol and type 2 diabetes and ensure that individuals get appropriate clinical management where needed, and (iii) to reduce inequalities in CVD health. In addition, people aged 65-74 are informed of the signs and symptoms of dementia, and sign-posted to memory clinics where appropriate [4]. A recent study highlighted the value of the NHSHC programme by demonstrating that patients who attend their NHSHC show demonstrable reductions in BMI, blood pressure and smoking incidence for 6 years afterwards [5], corroborating an earlier review which found that the programme can achieve small reductions in CVD risk [6].

Since April 2013, implementation of the programme has been the responsibility of local government (LG). Flexibility is permitted regarding how the programme is commissioned, although the tests, measurements and actions taken at certain risk factor thresholds are standardised to help ensure the safety, quality and effectiveness of the programme [4]. Substantial variation in programme delivery exists across LG, from the invitation process to the location of the checks (e.g., General Practitioner [GP] surgery, pharmacy etc.) to the healthcare professional carrying out the NHSHC.

Despite considerable variation in uptake rates across LG [7], there is little evidence for how these rates are influenced by differences in local programme delivery. This is an important area of focus given that ensuring a high percentage of those offered a NHSHC actually receive one is key to optimising the clinical and cost effectiveness of the programme [8]. Whilst there are no set targets for uptake, NHSHC funding allocations were originally modelled on an estimated uptake rate of $75 \%$ [8]. The national average uptake rate is currently $48.4 \%$ which means that just over half of invited patients do not attend their NHSHC [7]. Despite efforts, uptake of the NHSHC remains below optimum levels.

At present, the use of an invitation letter is the most common route for inviting eligible individuals for an NHSHC [9], and a standard template letter exists [7]. However, it is unclear how effective this method is compared to other invitation methods, and whether invitation mode varies in effectiveness for different groups of people. A review on general health checks [10] found that those least likely to attend were men on low incomes, those of low socio-economic status, the unemployed and the less well educated. Non-attenders also had a greater proportion of cardiovascular risk factors than attenders [10]. It is important to understand whether this pattern of attendance also holds for the NHSHC, and whether different groups of people are more likely to respond to certain interventions and invitation methods than others. The aim of this systematic review, therefore, is to highlight interventions and invitation methods that increase the uptake of $\mathrm{NHSH}$ Cs, and to identify whether the effectiveness of these interact with broader patient and contextual factors. Literature that investigated the impact of patient demographic and contextual factors, but did not explore the impact of invitation methods or interventions, was also consulted in order to understand wider trends in uptake and help to interpret the findings of this review. To the authors' knowledge there has been no systematic review published on this topic. Rapid reviews on similar topics have been completed by Cooper and Dugdill [11] and Usher-Smith and colleagues [6]. However the current paper is the first systematic literature review to report only high-quality evidence on interventions, invitation methods and patient and contextual characteristics that influence uptake of NHSHCs. In addition, no previous reviews have attempted to examine whether invitation methods and interventions vary in effectiveness by patient demographic characteristics. The overarching aim of conducting this systematic review is to contribute to evidence-based practice by translating evidence into current practice service delivery, and help steer the future direction of research.

\section{Method}

The Preferred Reporting Items for Systematic Reviews and Meta Analyses (PRISMA) 27 item checklist [12] and the Critical Appraisal Skills Programme Systematic Review checklist [13], were used to structure and scrutinise the systematic review. This systematic review was registered with PROSPERO on 22/02/2016 (CRD42016035626). The original aim specified in the protocol was to identify interventions and invitation methods in hard-to-reach groups specifically. Due to the lack of studies focused on the 
uptake of NHS HCs in hard to reach groups, it was decided to expand the focus of this systematic review to include all those eligible for an NHS HC and explore the patient characteristics associated with uptake.

\section{Study eligibility \\ Inclusion criteria}

The Patient-Intervention-Comparison-Outcome-Study (PICOS) framework [14] was used to develop eligibility criteria for the literature search strategy:

\section{Patients}

Eligible for a NHSHC (patients aged between 40 and 74 years with no existing diagnosis of heart disease, stroke, diabetes, kidney disease or high blood pressure).

\section{Intervention}

All studies that provided a clear description of the local implementation of the programme plus at least one of (i) the patient and or practice characteristics, (ii) invitation process, or (iii) an intervention implemented to encourage attendance at an NHSHC were included.

\section{Comparison}

Standard invitation method (for intervention studies), other types of invitation method (for studies comparing different existing methods if invitation), patients who do not attend NHSHCs (for studies investigating patient demographic characteristics).

\section{Outcome}

Uptake of or attendance at the NHSHC.

\section{Study type and design}

Only studies focused on NHSHCs were included in this review. Studies were required to have been published in 2009 onwards (as this is when the programme was implemented) and in English (as this is the only language spoken by the research team). This review intended to exclusively include randomised controlled trials since they are most able to support inferences of causality about interventions, but due to the limited number of studies, quasi-experimental research design trials were also included. Therefore, the following study designs were included: randomised controlled trials, observational cohort studies or cross-sectional studies, which may also be used to support inferences about causality.

\section{Exclusion criteria}

Any studies that were qualitative in design, a service evaluation or reported only subjective or self-reported outcomes were excluded. Any studies focused on children or individuals previously diagnosed with CVD or any interventions that focused on screening or disease-specific health checks other than NHSHC were excluded.

\section{Search strategy}

Between January 2015 and May 2015, a systematic review was conducted. This was repeated in August 2016 and then again in March 2018. A different list of databases was searched in March 2018 as a result of the lead author's completion of a university course and thus the termination of access to university library services (Table 1 ).

The Cochrane Library, Database of Abstracts of Reviews of Effects (DARE), Trip Database, NICE Evidence Services and PubMed Health were also searched at each time point to identify relevant systematic reviews. The reference list of review articles and all studies included within the review were also searched in order to find other potentially eligible studies. A hand search was carried out in recent journal editions.

The searches included terms selected to identify literature that (i) was relevant to the NHSHC specifically (e.g., "NHS Health Check" OR health check* OR (nhs and health check $\left.^{*}\right)$ ), (ii) investigated the impact of invitation methods and interventions (e.g., intervention* OR invit* OR offer* OR encourage ${ }^{*}$ ) and (iii) included uptake as an outcome measure (e.g., uptake OR attend* OR appointment*). See Additional file 1 for the full list of search terms used in this review and Additional file 2 for the hits by database.

\section{Study selection}

Study records (titles) were screened by one researcher (AKB 2015 \& 2016; LP 2018) in EndNote to identify articles for detailed abstract screening. One researcher (AKB 2015 \& 2016; LP 2018) selected suitable abstracts (or those which did not provide sufficient information for eligibility assessment) for full review. Full review involved two researchers (AKB \& BH 2015 \& 2016; AKB \& LP 2018) screening the full study text and populating a data extraction form to ensure the study met the inclusion criteria (Additional file 3). Figure 1 details the number of articles assessed at each stage. A list of studies excluded at the full text review stage and justifications is included in Additional file 4. The nine studies that met the inclusion criteria also underwent a quality assessment using an adapted tool (Additional file 5) specially developed to accommodate both randomised and nonrandomised studies. Two researchers reviewed the quality of selected studies independently (AKB \& BH 2015 \& 2016; AKB \& LP 2018) The checklist scores were reviewed, and then any discrepancies were identified discussed and resolved by referring to the research paper.

For the initial 2015 and 2016 reviews (in which seven papers were identified as eligible for inclusion) the interrater reliability was calculated using Cohen's kappa [15], which 
Table 1 Databases searched in 2016 and 2018

\begin{tabular}{ll}
\hline Searches up to August 2016 & March 2018 \\
\hline - Cochrane Library (Including Cochrane & - Ovid Medline \\
Database of Systematic Reviews and & - Ovid Embase \\
Cochrane & - Ovid Psycinfo \\
Central Register of Controlled Trials) & - EBSCO CINAHL \\
- EBSCOHOST (Including CINAHL Plus & - CDSR and CTR \\
with full text, Psych Info, Psych Articles & - SCOPUS \\
and MEDLINE) & - Google Scholar \\
- Ovid (including Embase) & \\
- SCOPUS & \\
- Web of Science & \\
- Google Scholar & \\
\hline
\end{tabular}

showed substantial agreement between the raters $(\mathrm{k}=0.772$, $p<0.0005$ ) [16]. The 2018 reviewers were in 100\% agreement over the additional two papers identified.

\section{Analysis}

A narrative synthesis is used to present the findings of this systematic review using the guidance from the Economic and Social Research Council [17]. A meta-analysis was not planned and has not been conducted as pooling results obtained from diverse non-randomised study-designs is not recommended [18].

\section{Results}

\section{Search results}

Nine-hundred-and-forty-four studies were identified after performing electronic de-duplication within and

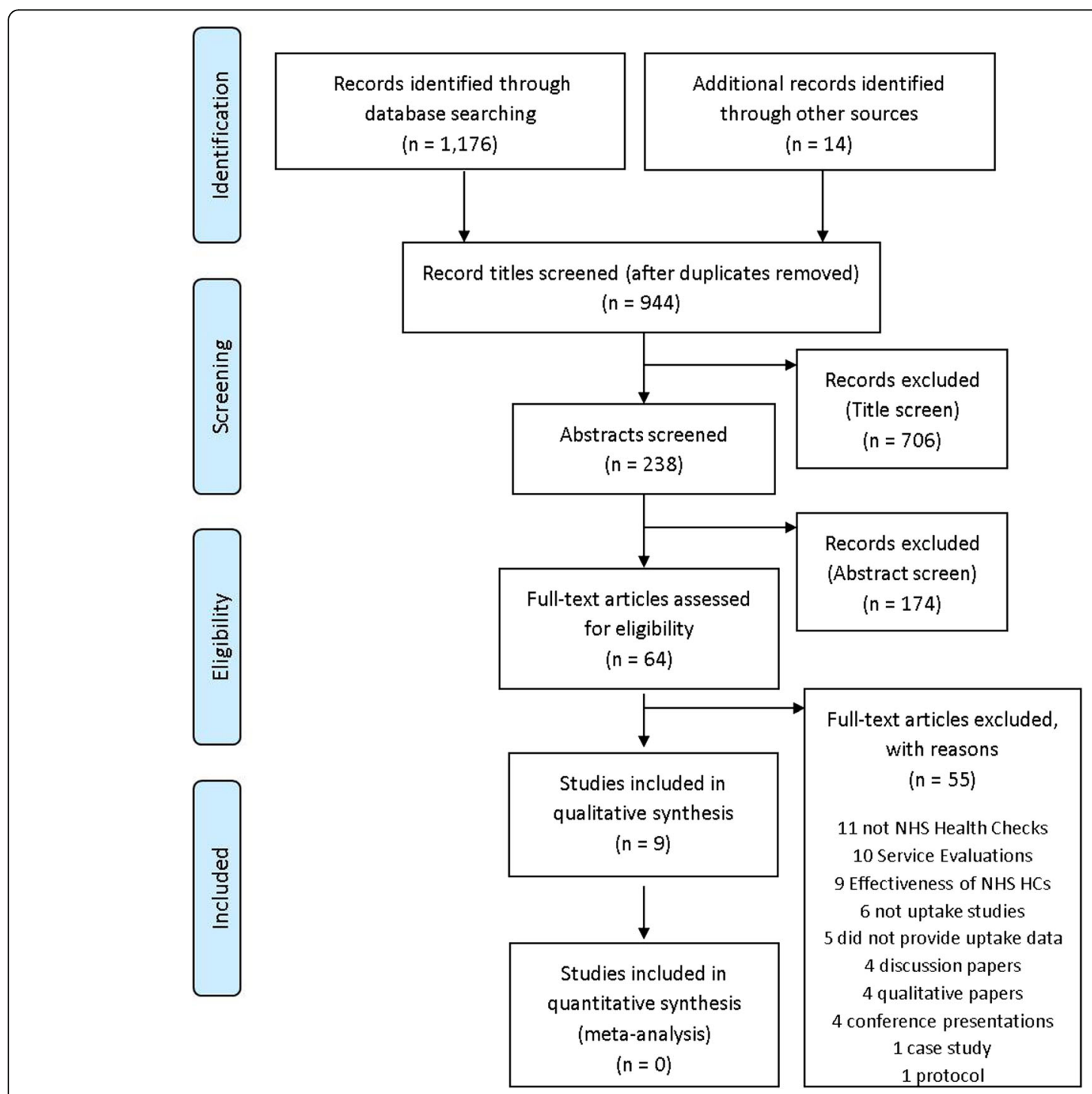

Fig. 1 Flow diagram of review process using the PRISMA (2009) template 
between each database (Additional file 2). After initial title screening, 238 titles and abstracts were identified as potentially relevant, of which 64 full papers were retrieved and assessed for eligibility (Fig. 1). A total of nine papers were included in the final review. No further studies were identified through the reference lists of the papers included, or subsequent hand searching.

\section{Study Characteristics \& Methodological Quality}

The nine included studies were conducted between 2011 and 2016, using data from 2008 to 2014. The sample sizes varied from 1380 patients [19] to 40,112 patients [20], and included between four [19, 21] and 38 GP Practices [22]. The length of studies (including the follow up period) varied from 6 months [23] to 4 years [22]. Six studies achieved a strong quality assessment rating $[9,20,21,23-25]$ and three achieved a moderate rating $[19,22,26]$. There were only two randomised controlled trials identified [21, 25], with the remaining studies consisting of one observational cohort [9] and six cross sectional studies [19, 20, 22-24, 26]. Table 2 summarises the study characteristics.

\section{NHSHC uptake \\ Invitation methods}

Two studies found that oral invitations (e.g., telephone invitations from the practice or opportunistic face-toface invitations at the practice) were more effective than written letter invitations, despite letters being the most commonly employed invitation type. Gidlow and colleagues [9] found that the majority of the five GP practices that they studied usually invited eligible patients to attend via an invitation letter $(72 \%$ of patients were invited in this way) but that the odds of individuals attending were three times greater when they were invited using a telephone/face-to-face approach compared to by letter alone (OR 2.87, 95\% CI =2.26-3.64). Similarly, Cook and colleagues [26] found that letters were the most common form of invitation but that face-to-face invitations had the highest overall uptake rate of $71.9 \%$ with uptake rates for both telephone (43\%) and letter (29.5\%) invitations markedly lower.

Only one study investigated whether invitation method effectiveness differed according to patient characteristics (although it is important to note that this was a cross-sectional study and did not randomise patients to different invitation methods) [26]. Given that letters were the most common method of invitation across the practices included in the study, it is unsurprising that those groups who had the lowest uptake rates overall also showed some of the lowest uptake rates for letter invitations specifically (e.g., 'Any Other White Background' males uptake $=19 \%, p<.001$, 'Any Other White Background' females uptake $=22 \%, p<.001$, and 'African' females $p=23 \%, p<.050)$. Face-to-face invitations were found to be most effective for 'White Irish' females (uptake $=93 \%, \mathrm{p}<.050$ ) and 'White British' male (uptake $=72 \%, p<.001$ ) and female patients (uptake $=70 \%$, $p<.001$ ), but the least effective method for inviting 'Bangladeshi' (uptake $=43 \%, p<.001$ ) and 'Pakistani' males (uptake $=47 \%, p<.050)$. Invitation by telephone was the least common method; however, where this method was used, it was most effective for 'Pakistani' patients of both genders (uptake $=100 \%, p<.010$ for both genders), 'White/Black Caribbean' females (uptake $=100 \%, p<.001$ ), 'White Irish' females (uptake $=96 \%, p<.001$ ) and 'Asian (Other)' females (uptake $=76 \%, p<.001$ ), but least effective for 'White British' females (uptake $=0 \%, p<.001$ ) and 'Any Other White Background' patients (uptake of 10 and $8 \%$ for males and females respectively, both $p<.001)$.

\section{Interventions on invitation methods}

Two studies were identified that tested interventions to enhance invitation methods with varying success. Sallis and colleagues [21] enhanced invitation letters using techniques from behavioural science including simplifying the text, personalisation, emphasising timelines (i.e., that the NHSHC was "due") and providing patients with a space to write down the time and date of their appointment. The odds of attending a NHSHC appointment were $26 \%$ higher for patients receiving an enhanced invitation letter compared to patients receiving the control letter $(\mathrm{AOR}=1.26,95 \%$ $\mathrm{CI}=1.09-1.47, p<.001)$. Uptake in the intervention arm was $33.5 \%$ compared to $29.3 \%$ in the control arm, an absolute difference of $4.2 \%$ and a relative difference of $14.3 \%$ in NHSHC attendance. However, McDermott and colleagues [25] found that mailing patients a Question-Behaviour Effect questionnaire (with or without an incentive for returning the questionnaire) 1 week before invitation letters did not significantly improve uptake in the intervention groups compared to the control group. These researchers found that uptake was higher for intervention group individuals who returned the questionnaire, but when examining the intervention group as a whole (i.e., in an intention to treat analysis), there was no significant effect of including a questionnaire $(p=.070)$ or a questionnaire plus incentive $(p=.054)$ compared to controls. Less than a quarter of participants returned the questionnaire and those who returned the questionnaires were more likely to be female, in older age groups, and have lower levels of deprivation. Interestingly, all of these demographic factors are associated with higher uptake of health checks which is discussed in the following section. Neither of the trials investigating interventions to improve uptake explored whether the effectiveness of the interventions interacted with patient demographic characteristics. 


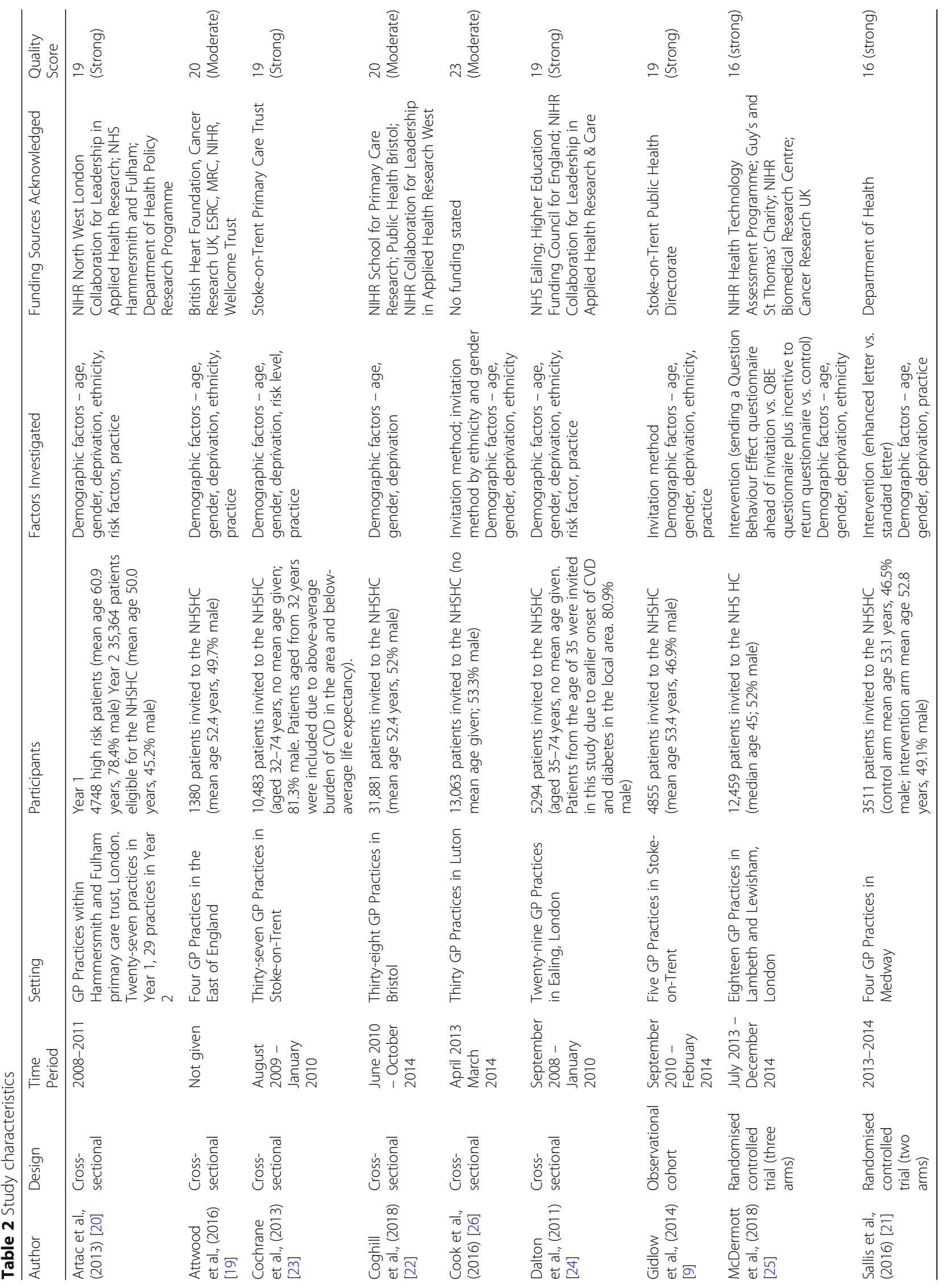




\section{Socio-demographic factors on uptake of NHSHCs Age}

All studies found that older patients were more likely to attend than younger patients (Table 3). This finding was consistent, regardless of whether studies tested the effects of age in increments of years $[9,19]$ or decades [21], or whether broader age groups were tested [20]. In addition, Dalton and colleagues found a significant interaction between age and gender, with women in the youngest age-group (35-54 years) more likely to attend than men of the same age-group [24].

\section{Gender}

The majority of studies found that uptake was highest for female patients (Table 4). Two studies both found that female patients were $50 \%$ more likely to attend than male patients [19, 21] (although one of these found that this association was no longer significant once practice had been added to the adjusted model [19]) while another found a similar increase of $47 \%$ likelihood for female patients [9]. Artac and colleagues found higher uptake among females when examining data from Year 2 when all eligible patients were invited for a NHSHC, but no gender difference was found in Year 1, when only high-risk patients were invited, $78.4 \%$ of whom were male [20]. In contrast to the majority of studies, Cochrane and colleagues [23] found lower uptake amongst female patients. Finally, as noted earlier, another study found that there was a significant interaction effect between age and sex, with women in the youngest age group being more likely to attend the NHSHC than men [24].

Table 3 Study findings on impact of age on NHSHC uptake. AOR=adjusted odds ratio. $\mathrm{OR}=$ odds ratio. $\mathrm{Cl}=\mathrm{confidence}$ intervals UR = uptake rate

\begin{tabular}{ll}
\hline Study & Findings \\
\hline Artac et al., $2013[20]$ & $55-64$ age-group vs. 40-54 age-group \\
& (baseline): AOR=1.34, 95\% Cl=1. \\
& $11-1.61, p<.050$ \\
& $65-74$ age-group vs. 40-54 age-group \\
& (baseline): AOR $=2.05,95 \% \mathrm{Cl}=1.67-2.52$, \\
& $p<.010$
\end{tabular}

Attwood et al., 2016 [19]

Cochrane et al., 2013 [23]

Coghill et al., 2018 [22]

Cook et al., 2016 [26]

Dalton et al., 2011 [24]

Gidlow et al., 2014 [9]

McDermott et al., 2018 [25]

Sallis et al., 2016 [21]
$5 \%$ increase in likelihood of uptake with each additional year in age: $\mathrm{OR}=1.05$, $95 \% \mathrm{Cl}=1.04-1.07, p<.010$

Overall effect of age-group: $\mathrm{OR}=1.64$, $95 \% \mathrm{Cl}=1.51-1.77, p<.001$

Overall effect of age $p<.001$ $50-59$ years vs. $\leq 49$ years (baseline): $\mathrm{AOR}=1.36,95 \% \mathrm{Cl}=1.21-1.53$ 60-69 years vs. $\leq 49$ years (baseline): $\mathrm{AOR}=2.19,95 \% \mathrm{Cl}=1.80-2.68$ $70+$ years vs. $\leq 49$ years (baseline): $\mathrm{AOR}=2.53,95 \% \mathrm{Cl}=1.89-3.39$

Highest uptake in 65-69 (male uptake $=71 \%$, $p<.001$, female uptake $=62 \%, p<.001)$ and $70-74$ age-groups (male uptake $=68 \%$, $p<.001$, female uptake $=80 \%, p<.001$ )

55-64 age-group vs. 35-54 age-group (baseline): $\mathrm{AOR}=1.74,95 \% \mathrm{Cl}=1.34-2.25$, $p<.001$

65-74 age-group vs. 35-54 age-group (baseline): $\mathrm{AOR}=2.27,95 \% \mathrm{Cl}=1.47-3.50$, $p<.001$

Significant age $x$ gender interaction; women in the youngest age-group (35-54 years) more likely to attend than men in the same age category: $\mathrm{AOR}=1.71,95 \% \mathrm{Cl}=1.03-2.85, p=.037$

$4 \%$ increase in likelihood of uptake with each additional year of age: $\mathrm{OR}=1.04,95 \% \mathrm{Cl}=1.03-1.04$, $p<.001$

60-74 age group vs. 40-59 age-group (baseline): $\mathrm{OR}=1.43,95 \% \mathrm{Cl}=1.20-1.71, p<.001$

$62 \%$ increase in likelihood of uptake with every additional 10 years in age: $\mathrm{AOR}=1.62,95 \% \mathrm{Cl}=1.50-1.75$, $p<.010$ 
Table 4 Study findings on impact of gender on NHSHC uptake. AOR=adjusted odds ratio. OR= odds ratio. $\mathrm{Cl}=$ confidence intervals.

\begin{tabular}{|c|c|}
\hline Study & Findings \\
\hline Artac et al., 2013 [20] & $\begin{array}{l}\text { Year } 1 \text { (high-risk only) } \\
\text { Female vs. male (baseline): } \mathrm{AOR}=0.80, \\
95 \% \mathrm{Cl}=0.67-0.94 p<.010 \\
\text { Year } 2 \text { (all eligible patients) } \\
\text { Female vs. male (baseline): } \mathrm{AOR}=1.27 \\
95 \% \mathrm{Cl}=1.20-1.35, p<.010\end{array}$ \\
\hline Attwood et al., 2016 [19] & $\begin{array}{l}\text { Unadjusted model } \\
\text { Female vs. male (baseline): } \mathrm{OR}=1.50 \\
95 \% \mathrm{Cl}=1.16-1.95, p<.050 \\
\text { Model adjusted for GP surgery effects } \\
\text { Female vs. male (baseline): AOR }=1.29 \\
95 \% \mathrm{Cl}=0.95-1.76, p>.050\end{array}$ \\
\hline Cochrane et al., 2013 [23] & $\begin{array}{l}\text { Female vs. male (baseline): } \mathrm{OR}=0.70 \text {, } \\
95 \% \mathrm{Cl}=0.58-0.84, p<.001\end{array}$ \\
\hline Coghill et al., 2018 [22] & $\begin{array}{l}\text { Male vs. female (baseline): } \mathrm{AOR}=0.73 \text {, } \\
95 \% \mathrm{Cl}=0.67-0.80, p<.001\end{array}$ \\
\hline Cook et al., 2016 [26] & $\begin{array}{l}\text { Female uptake rate }=50 \% \text {; male uptake } \\
\text { rate }=38 \%, p<.001\end{array}$ \\
\hline Dalton et al., 2011 [24] & $\begin{array}{l}\text { Significant age } x \text { gender interaction; } \\
\text { women in the youngest age-group (35-54 years) } \\
\text { more likely to attend than men in the same age } \\
\text { category: AOR }=1.71,95 \% \mathrm{Cl}=1.03-2.85, p=.037\end{array}$ \\
\hline Gidlow et al., 2014 [9] & $\begin{array}{l}\text { Female vs. male (baseline): } \mathrm{OR}=1.47 \text {, } \\
95 \% \mathrm{Cl}=1.30-1.68, p<.001\end{array}$ \\
\hline McDermott et al., 2018 [25] & $\begin{array}{l}\text { Male vs. female (baseline): } \mathrm{AOR}=0.74 \\
95 \% \mathrm{Cl}=0.69-0.80, p<.001\end{array}$ \\
\hline Sallis et al., 2016 [21] & $\begin{array}{l}\text { Female vs. male (baseline): } \mathrm{AOR}=1.50 \text {, } \\
95 \% \mathrm{Cl}=1.29-1.74, p<.010)\end{array}$ \\
\hline
\end{tabular}

\section{Deprivation}

Where a significant effect of deprivation on uptake was found, the majority of studies reported that this was due to lower uptake in more deprived groups $([9,21,25,26]$; see Table 5). However, in other studies, this relationship was dependent on whether analyses were adjusted for other factors or not. For example, in unadjusted analyses, Cochrane and colleagues [23] found a significant pattern of decreasing uptake as practice-level deprivation increased. However, deprivation was no longer significant when analyses were adjusted for gender, age, risk category and practice size.

Meanwhile, Attwood and colleagues [19] found that the direction of the relationship between deprivation and uptake depended on whether or not analyses were adjusted for other predictors (e.g., age, gender, ethnicity, GP practice), with adjusted analyses revealing the same pattern of lower uptake in the most deprived groups as seen in many other studies, but unadjusted analyses revealing higher uptake in more deprived groups. Similarly, in adjusted analyses, Artac and colleagues [20] found higher uptake amongst patients living in deprived areas in Year 2 of the programme only (when all eligible patients were invited; no effect of deprivation was found when only high-risk patients were invited). Two studies [22, 24] found no significant effect of deprivation on uptake.

\section{Ethnicity}

Findings on ethnicity presented a mixed picture across the studies: some found that attendance was significantly higher in certain ethnic groups and others found that uptake did not differ by patient ethnicity. Uptake was found to be higher in patients from Asian groups (including South Asian and Asian-Indian groups) [20, 24-26]; Black groups (including Black African and Black Caribbean groups) [20, 25]; and mixed ethnicity groups $[24,25]$. Contrary to other findings, Cook and colleagues [26] found that amongst females, uptake rates were lowest for Black African patients, however they observed higher uptake rates amongst Black Caribbean patients of both genders in line with other studies reporting high uptake amongst Black patients. In contrast, two studies found that there was no significant difference by ethnic group $[9,19]$. Detailed findings on ethnicity are presented in Table 6 below.

\section{Medical and lifestyle risk}

Studies' conceptualisation of risk varied, with some classifying it as a medical risk (e.g., family history of CVD) and others including lifestyle factors (e.g., smoking status; see 
Table 5 Study findings on impact of deprivation on uptake. Quintile/tertile 1 refers to the most deprived group. Note that some studies $[19,22,25,26]$ coded deprivation so that quintile/tertile 1 referred to the least deprived group, but that this has been reversed for the current narrative synthesis in order to match other studies' reporting standards and enhance comparability across studies. $\mathrm{IMD}=$ Index of Multiple Deprivation, $\mathrm{AOR}=$ adjusted odds ratio. $\mathrm{OR}=$ odds ratio. $\mathrm{Cl}=$ confidence intervals

Study
Artac et al., 2013 [20]

Attwood et al., 2016 [19]

Cochrane et al., 2013 [23]

Coghill et al., 2018 [22]

Cook et al., 2016 [26]

Dalton et al., 2011 [24]

Gidlow et al., 2014 [9]

McDermott et al., 2018 [25]

Sallis et al., 2016 [21]

\section{Findings}

Year 1 analyses (high-risk patients only) IMD Tertile 3 vs. 1: $A O R=0.84$,

$95 \% \mathrm{Cl}=0.69-1.01, p>.050$

IMD Tertile 2 vs. 1: $\mathrm{AOR}=0.94$,

$95 \% \mathrm{Cl}=0.79-1.13, p>.050$

Year 2 analyses (all eligible patients)

IMD Tertile 3 vs. 1: $A O R=0.80$,

95\% Cl = 0.73-0.87, $p<.010$ IMD

Tertile 2 vs. $1: \mathrm{AOR}=0.84$,

$95 \% \mathrm{Cl}=0.78-0.90, p<.010$

Unadjusted analyses

IMD Quintile 2 vs. 5: OR=2.17

$95 \% \mathrm{Cl}=1.39-3.38, p<.010$

IMD Quintile 1 vs. 5: $\mathrm{OR}=2.90$,

$95 \% \mathrm{Cl}=1.84-4.58, p<.010$

Adjusted analyses

IMD Quintile 2 vs. 5: $A O R=0.37$,

$95 \% \mathrm{Cl}=0.18-0.67, p<.050$

IMD Quintile 1 vs. 5: $A O R=0.42$,

$95 \% \mathrm{Cl}=0.20-0.88, p<.050$

Lowest attendance in tertile 3

(attendance rate $=42.6 \%, p<.050$ )

Highest attendance in tertile 1

(attendance rate $=48.4 \%, p<.050$

Deprivation was no longer significant

when analyses were adjusted for gender, age, risk category and practice size $\mathrm{AOR}=1.12,95 \% \mathrm{Cl}=0.96-1.30$

Non-significant effect of deprivation on uptake $(p=.053)$

Lowest uptake in Quintile 1 with uptake rates of 0.31 and 0.38 for males and females respectively, $p<.001$

Highest uptake in the Quintile 5, with uptake rates of 0.53 and 0.60 respectively, $p<.001$

No significant effect of deprivation $(p>.050)$

IMD Quintile 5 vs. 1: OR $=1.59,95 \%$

$\mathrm{Cl}=1.23-2.05, p<.001$

IMD Quintile 4 vs. 1: OR $=1.30,95 \%$

$\mathrm{Cl}=1.06-1.61, p=.014$

IMD Quintile 3 vs. 1: OR $=1.24,95 \%$

$\mathrm{Cl}=1.03-1.49, p=.022$

IMD Quintile 2 vs. 1: OR = 1.11, 95\%

$\mathrm{Cl}=0.87-1.43, p=.395$

Overall effect of deprivation $p=.008$

IMD Quintile 4 vs. 1: $A O R=2.78,95 \%$

$\mathrm{Cl}=1.87-4.12, p<.001$

IMD Quintile 3 vs. 1: $A O R=1.15,95 \%$

$\mathrm{Cl}=0.95-1.39, p=.156$

IMD Quintile 2 vs. 1: $A O R=1.09,95 \%$

$\mathrm{Cl}=0.95-1.24, p=.214$

(Note, no data was collected from

Quintile 5 in this study)

IMD Quintile 5 vs. 1: $A O R=1.61,95 \%$

$\mathrm{Cl}=1.14-2.26, p<.010$

All other comparisons against Quintile

1 (baseline) $p>.010$ 
Table 6 Summary of findings on ethnicity across studies. AOR = adjusted odds ratio. OR= odds ratio. $\mathrm{Cl}=$ confidence intervals. UR= uptake rate

\begin{tabular}{ll}
\hline Study & Findings \\
\hline Artac et al., 2013 [20] & In Year 2, higher in patients of South \\
& Asian (AOR=1.50, $95 \% \mathrm{Cl}=1.25-1.78$, \\
& $p<.010)$ and Black ethnicity $(\mathrm{AOR}=1.58$, \\
& $95 \% \mathrm{Cl}=1.43-1.75, p<.010)$ and Others \\
& $(\mathrm{AOR}=1.16,95 \% \mathrm{Cl}=1.07-1.25, p<.010)$ \\
& where White is baseline.
\end{tabular}

Attwood et al., 2016 [19]

Cook et al., 2016 [26]

Dalton et al., 2011 [24]

Gidlow et al., 2014 [9]

McDermott et al., 2018 [25]

No difference between White ethnicity vs. Other ethnicity $(\mathrm{OR}=0.59,95 \%$ $\mathrm{Cl}=0.21-1.57)$

Higher uptake amongst Mixed White and Asian males (UR $=0.91,95 \% \mathrm{Cl}=0.66-0.99$, $p<.001)$, Caribbean males (UR $=0.69,95 \%$ $\mathrm{Cl}=0.62-0.76, p<.001)$, Chinese males $(\mathrm{UR}=0.67,95 \% \mathrm{Cl}=0.45-0.84, p<.010)$, Chinese females (UR $=0.93,95 \%$ $\mathrm{Cl}=0.72-0.99, p<.001)$, White/Black Caribbean females (UR $=0.77,95 \%$ $\mathrm{Cl}=0.63-0.87, p<.001)$, White Irish females $(\mathrm{UR}=0.72,95 \% \mathrm{Cl}=0.64-0.80, p<.001)$, and Black Caribbean females (UR $=0.71,95 \%$ $\mathrm{Cl}=0.64-0.77, p<.001)$.

Lower uptake amongst any other white patients (male UR $=0.27,95 \% \mathrm{Cl}=0.24-0.30, p<.001$; female UR $=0.35,95 \% \mathrm{Cl}=0.31-0.38, p<.001)$ and Black African females (UR $=0.42,95 \%$ $\mathrm{Cl}=0.37-0.47, p<.010)$.

When compared to White British patients, uptake higher in patients of South Asian $(\mathrm{AOR}=1.71,95 \% \mathrm{Cl}=1.29-2.27, p<.001)$ and mixed ethnic backgrounds $(\mathrm{AOR}=2.42$, $95 \% \mathrm{Cl}=1.50-3.89, p=.015)$.

No difference between White, Mixed, Asian, Black or Other ethnicity groups $\left(X^{2}=0.769\right.$, $p=.380)$

Higher uptake amongst Asian (OR $=2.03$, $95 \% \mathrm{Cl}=1.63-2.67, p<.001)$, African/Caribbean $(\mathrm{OR}=2.15,95 \% \mathrm{Cl}=1.86-2.49, p<.001)$ and mixed $(\mathrm{OR}=3.09,95 \% \mathrm{Cl}=2.07-4.62, p<.001)$ ethnicity groups compared to White patients.

Table 7). Cochrane and colleagues [23] found a nonsignificant trend towards decreased likelihood of attendance for patients deemed at higher risk of CVD. Conversely, Artac and colleagues found that risk factors such as presence of non-CVD comorbidities and family history of coronary heart disease were both significant predictors for increased uptake across both years of the programme, whereas smoking status was a significant predictor for decreased uptake across both years [20]. A non-significant trend for lower uptake amongst smokers was found by Dalton and colleagues [24]. This suggests that the association between risk level and uptake may vary depending on the definition of risk and whether risk is assessed based on medical history or lifestyle factors (Table 7 ).

\section{Practice differences}

Interestingly, all studies reporting on GP practice found significant variance in uptake between practices (Table 8)
$[9,19-21,23,24]$, however, it was not always possible to discern the reasons for this. Two studies found some evidence that practice list size impacted NHSHC attendance $[20,24]$, however the direction of these effects was different, and Cochrane and colleagues [23] found that practice size was not significantly related to uptake. Gidlow and colleagues [9] found that NHSHC attendance did not vary by distance to practice from the patient's home. No other specific practice-level factors were reported.

\section{Discussion}

This review aimed to identify invitation methods and interventions that increased uptake of NHSHCs, and to explore whether the effectiveness of these varied by patient demographic characteristics and contextual factors. The studies included all achieved a high or moderate quality rating, suggesting that risk of bias is 
Table 7 Study findings on impact of risk factors on NHSHC uptake. AOR=adjusted odds ratio. OR= odds ratio. $\mathrm{Cl}=$ confidence intervals. UR = uptake rate

\begin{tabular}{|c|c|}
\hline Study & Findings \\
\hline Artac et al., 2013 [20] & $\begin{array}{l}\text { Impact of comorbidities (medical risk) } \\
\text { Year } 1 \mathrm{AOR}=1.53,95 \% \mathrm{Cl}=1.31-1.80, p<.010 \\
\text { Year } 2 \mathrm{AOR}=1.75,95 \% \mathrm{Cl}=1.64-1.87, p<.010 \\
\text { Impact of family history (medical risk) } \\
\text { Year } 1 \mathrm{AOR}=2.49,95 \% \mathrm{Cl}=2.15-2.90, p<.010 \\
\text { Year 2 } \mathrm{AOR}=2.01,95 \% \mathrm{Cl}=1.87-2.16, p<.010 \\
\text { Impact of smoking status (lifestyle risk) } \\
\text { Year 1 } \mathrm{AOR}=0.71,95 \% \mathrm{Cl}=0.61-0.83, p<.010 \\
\text { Year 2 } \mathrm{AOR}=0.83,95 \% \mathrm{Cl}=0.77-0.90, p<.010 \text { ) }\end{array}$ \\
\hline Cochrane et al., 2013 [23] & $\begin{array}{l}\text { Risk category (combination of medical and lifestyle risk) } \\
\mathrm{OR}=0.90,95 \% \mathrm{Cl}=0.80-1.02, p>.050 \text { and }<.100\end{array}$ \\
\hline Dalton et al., 2011 [24] & $\begin{array}{l}\text { Smoking status (lifestyle risk) } \\
\text { Yes vs. no (baseline): } \mathrm{AOR}=0.88,95 \% \mathrm{Cl}=0.75-1.92 \\
p=.097\end{array}$ \\
\hline
\end{tabular}

low. Overall, it was possible to identify which invitation methods and patient demographic characteristics were associated with increased uptake, but very little evidence was available with regards to how patient demographic characteristics interact with invitation methods and interventions to increase uptake, representing a significant limitation of the existing literature. Letters are the most widely used invitation method within the NHSHC programme [9, 26] and our findings revealed that compared to telephone and

Table 8 Study findings on the impact of practice and specific practice-level factors on NHSHC uptake. AOR=adjusted odds ratio. $\mathrm{OR}=$ odds ratio. $\mathrm{Cl}=$ confidence intervals. $\mathrm{UR}=$ uptake rate

\begin{tabular}{|c|c|}
\hline Study & Findings \\
\hline Artac et al., 2013 [20] & $\begin{array}{l}\text { Practice List Size Year } 2 \text { (all eligible patients) } \\
>10,000 \text { vs. }<6000 \text { (baseline): AOR }=6.05, \\
95 \% \mathrm{Cl}=0.84-43.3, p<.010 \\
\text { Unexplained variance in models was interpreted } \\
\text { as attributable to unmeasured practice factors } \\
\text { Year } 1 \text { unexplained variance }=19.4,95 \% \\
\mathrm{Cl}=15.2-24.4 \% \\
\text { Year } 2 \text { unexplained variance }=37.3,95 \% \\
\mathrm{Cl}=30.6-44.6 \%\end{array}$ \\
\hline
\end{tabular}

Attwood et al., 2016 [19]

Cochrane et al., 2013 [23]

Dalton et al., 2011 [24]
Significant variance in uptake by practice $X_{2}^{2}=74.61, p<.005$

Adjusting analyses for GP Practice had a substantial effect on the strength and direction of associations between socio-demographic variables (specifically gender and IMD quintile; see relevant sections above) and uptake.

Practice Size

$\mathrm{AOR}=1.03,95 \% \mathrm{Cl}=0.88-1.20, p>.100$

Variance accounted for by individual practices $=12.7 \%, p<.001$

Practice List Size

$<3000$ vs. 3000-5999 (baseline):

$\mathrm{AOR}=2.53,95 \% \mathrm{Cl}=1.09-5.84, p=.030$

$\geq 6000$ vs. 3000-5999 (baseline): $\mathrm{AOR}=0.79,95 \% \mathrm{Cl}=0.33-1.88, p=.599$

Variance in models accounted for by practice $=28 \%(\mathrm{VPC}=0.28)$

Variation in uptake by practice $X^{2}=336.9, p<.001$

Variation in uptake by distance to practice $X^{2}=0.478, p=.924$

Of the five practices studied, one (used as baseline in analyses) had significantly higher uptake rates than all others (all $p<.010$ ). 
opportunistic face-to-face invitations, they were the least effective at encouraging uptake. Given this, it is perhaps unsurprising that the only two interventions identified in our search both focused on enhancing letter invitation techniques. Sallis and colleagues [21] made changes to the existing national template letter using a behavioural insights approach, and found an increase in uptake for those who received the intervention letter. Two recent studies (one under review, one published after our searches were conducted) also found significant, positive impacts of enhancing invitation letters using insights from behavioural science; one study used techniques such as message simplification and encouraging behavioural planning in the letter to increase uptake [27] whilst another enhanced uptake by either discussing sunk costs in the letter (telling patients that funding had already been set aside for their appointment) or providing counterarguments against common reasons for not attending (e.g., by telling patients that lifestyle factors can have an impact on CVD risk even in the presence of a family history of disease [28].

Meanwhile, McDermott and colleagues [25] found that posting a Question-Behaviour Effect questionnaire (with or without a financial incentive to encourage the questionnaire's return) ahead of invitation letters did not have a significant effect on uptake, possibly due to low rates of questionnaire return. This technique has been successful in other areas (e.g., general health checks, influenza vaccinations [29], so its failure to increase NHS Health Check uptake highlights the importance of trialling interventions applied to new areas, even when they have been successful in similar fields. Recently, Gold and colleagues found that providing an enhanced leaflet alongside invitation letters also failed to significantly impact uptake rates [30]. Together, these findings suggest that making enhancements to the invitations directly (instead of to any accompanying materials) may be the most effective strategy to improve uptake.

The results of this review with regards to effectiveness of invitation method are reflected elsewhere in the literature. For example, a recent study (published outside of our search dates) also found that uptake was significantly higher after telephone invitations (estimated effect was an additional 180 NHSHCs attended for every 1000 patients) compared to letters personalised to patients' CVD risk (estimated additional 40 NHSHCs per 1000 patients) and the standard invitation letter in use at the time [31]. Letters are a low-cost invitation method and have previously been recommended due to a lack of evidence for the cost-effectiveness of other methods [32]; however there is evidence that the high uptake rates associated with telephone invitations may make this method more cost-effective [31].
There are a couple of possible explanations for this relative effectiveness at encouraging uptake. A recent review and qualitative synthesis identified a number of reasons for not taking up the offer of a NHSHC, including misunderstanding the purpose of the appointment and the prioritisation of other daily demands [33]. It is possible that speaking directly to patients through telephone and faceto-face invitations allows these barriers to be discussed and broken-down, while also removing some of the steps in the appointment booking process. Recent work examining telephone outreach approaches revealed that patients appreciated being able to ask questions and receive immediate answers, and that patients also appreciated the immediacy of being able to book an appointment during the outreach call, stating that they might not have gotten around to booking an appointment or even reading the letter in the first place [34]. .However, it is also worth noting that since the studies reviewed here were conducted, the national template letter has been updated based on a series of studies that successfully increased NHSHC uptake by enhancing this letter [21, 27] (one of which was reviewed here [21];), meaning that comparisons of oral methods against written methods may yield different outcomes in future research.

The study by Cook and colleagues (which was the only one identified exploring invitation method effectiveness by demographic characteristics) found that the effectiveness of invitation methods varied by patient ethnicity (although it should be noted that this was not a randomised study and while it achieved a moderate rating in the quality assessment, it was only one point away from being scored as low quality) [26]. For example, whilst face-to-face invitations were most effective for White British patients, they were least effective for Bangladeshi and Pakistani males; conversely, telephone invitations were most effective for Pakistani patients and least effective for White British females and those identifying as any other white ethnicity. While the reasons for this differential success were not explored in the study by Cook and colleagues [26], a recent qualitative investigation of a telephone outreach intervention for deprived communities and ethnic minority groups found that patients appreciated receiving a proactive invitation by telephone, and particularly valued calls when the caller was someone with whom they could culturally identify and, in cases of language barriers, communicate in their first language [34]. This corroborates qualitative evidence from other health programmes which found that difficulties with reading written materials in English posed a barrier for South Asian patients [35]. It is not clear whether the GP practices included in the study by Cook and colleagues contacted patients through a similar outreach programme targeted 
towards ethnic minority groups, however these findings provide a starting point both for future research and current practice. In particular, where telephone/ face-to-face invitations may not be feasible or affordable for practices to deliver to all eligible patients, identifying specific patient groups (i.e., those who are high risk or who are likely to be particularly responsive to this type of invitation) for telephone invitations could be an appropriate strategy for allocating resources effectively.

An alternative use of resources could be to use telephone invitations as a follow-up reminder for patients who do not respond to the initial letter; evidence from increasing attendance at hospital appointments has shown the effectiveness of reminder messages [36], suggesting that reminder phone calls could be a successful strategy. Research in this area is also testing the effectiveness of text messages, which are cheaper per patient to send than phone calls; one study found that sending a reminder text as a follow-up to a letter increased NHSHC uptake [27]. These results show how different forms of telephone contact can improve NHSHC uptake. Future research could therefore investigate the costeffectiveness of text message reminders as a method to engage those groups with lowest attendance rates. Research could also investigate if these methods could minimise the difference in attendance by demographic factors, or whether logistical issues (e.g., related to keeping updated records of patient phone numbers) would limit the effectiveness of this method.

In terms of the impact of demographic factors on uptake, the review revealed that all studies found higher NHSHC attendance in older patients and the majority of studies found that females were more likely to attend than males. This latter finding is concerning as men may be at greater risk of CVD than women (for example, over $70 \%$ of high-risk patients were male in one study [20]). However, there was some variation, with two studies finding that male patients were more likely to attend than females, and another finding that the beneficial effect of being female was no longer significant once GP surgery had been accounted for in the model, suggesting that the effect of gender on uptake is not as reliable as the effect of age. As suggested by Usher-Smith and colleagues [6], the finding of an interaction between age and gender in one study (where females in the youngest age category were more likely to attend than males, but no difference was detected for older patients) [24] may provide an explanation for this variability across studies, in that younger female patients are more likely to take up a check than their male counterparts but that this increased likelihood attenuates with age.

There was also evidence that the direction of the association between level of risk and uptake varied according to the specific risk factor under investigation, with medical risk (e.g., family history) being associated with higher uptake and lifestyle risk (e.g., smoking status) being associated with lower uptake. This complexity is an important finding and warrants further investigation as patients at high risk of CVD are those who services want and need to engage with most urgently. These results mirror similar findings on engagement with healthcare services whereby those demonstrating high risk are less likely to attend health appointments [10,37].

There is also a mixed picture in relation to ethnicity across the studies. For example, three studies reviewed here found that attendance was significantly higher in patients from South Asian, Asian and Black backgrounds $[20,24,25]$, while another found that uptake was highest in Asian-Indian, Black-Caribbean and White British groups, but lowest in Black African groups [26]. In addition, others reported that uptake did not differ by patient ethnicity [9, 19]. Further complication arose from the finding that many of the studies showed high levels of missing ethnicity data [9, 20, 24-26], with Dalton and colleagues [24] finding that ethnicity data was missing for $31.8 \%$ of invited patients and $37.9 \%$ of NHSHC attendees. Whilst Artac and colleagues [20] specified that missing ethnicity data was sometimes due to patients being unwilling to disclose this information, Cook and colleagues [26] commented that high levels of missing data were due to GP practices failing to routinely update and audit their records, with Coghill and colleagues also commenting that poor recording of ethnicity by practices precluded the possibility of investigating the association between ethnicity and attendance in their study [22]. The complexity in uptake patterns revealed by this review highlights the importance of accurate and detailed ethnicity recording when investigating NHS Health Check uptake, as patients from specific ethnicities (e.g., Black African, Any Other White) can demonstrate different uptake patterns to the broader group level (e.g., Black, White).

The effect of deprivation on uptake of NHSHCs also varied across the studies and seemed to be influenced in some cases by whether or not analyses were adjusted for other predictor variables. Unfortunately, no studies investigated whether the impact of different invitation methods varied by deprivation levels. It is therefore not possible to assess which invitation strategies could be best used to engage those from the most deprived quintiles, who (as the majority of studies reviewed here found) were less likely to take up their NHSHC compared to patients within the least deprived quintiles. These findings dovetail with the findings of a large scale report that revealed that more affluent patients were more likely to respond to the invitation than less affluent patients (although overall coverage was higher among 
those from deprived communities [38]). This, alongside consistent evidence for variation between healthcare practices, demonstrates how important local context may be on the uptake of NHSHCs and indeed all health services. It is possible that the variation in uptake between individual practices may be a result of high correlations between individual practices and deprivation levels (e.g. [28],), a suggestion that is supported by the finding that adding GP practice to analyses impacted the relationship between deprivation and uptake in one study [19]. Indeed, other studies in this review (e.g. [21],) used the practice's postcode as a measure of IMD for patient uptake, demonstrating how interlinked these factors are in research. However, the importance of considering other GP practice-specific factors when exploring reasons for uptake should not be ignored. This includes factors pertaining to the local delivery of the programme, such as the invitation process and whether opportunistic checks are conducted. Qualitative work in this area can also help to shed light on practice-specific factors that impact uptake, such as a lack of convenient appointment times and difficulty booking due to waiting lists $[39,40]$.

\section{Limitations of the review}

One limitation is that in this instance, it was not possible to conduct a meta-analysis due to the substantial variation in the design of included studies [18]. An additional limitation is that this systematic review focused exclusively on literature on NHSHCs, possibly excluding relevant research from other programmes. However, this decision was made because, as far as the authors are aware, there are no other population-level preventative health check programmes with the same scope as the $\mathrm{NHSHC}$, and there have been an increasing number of requests from LG for evidence on what works to increase uptake specifically for NHSHCs.

The search strategy identified many studies investigating interventions to increase uptake of NHSHCs, especially within hard to reach groups, however these were often conducted as service evaluations by local areas (e.g. [41],). Unfortunately, due to the designs of these studies, they could not be included in this systematic review, but they may hold important information about implementation locally. If local government were to collaborate with academics to utilise more robust research designs and facilitate more vigorous evaluations, this would enable more evidence to be collected more easily about programmes such as NHSHCs to help inform best practice. Increasingly, there are funding opportunities available for implementation research and academics keen to find local areas to test promising interventions. A recent strategy published by Public Health England's Behavioural Insights team (PHEBI) makes steps towards this goal as it was developed with the aim of encouraging a greater integration of traditionally academic behavioural and social science disciplines into public health practice [42].

Finally, this review only included studies with quantitative measures of uptake and did not investigate qualitative work on the experiences of patients and practitioners in regards to NHSHC invitation processes, NHSHC appointments, and potential barriers and facilitators to uptake. A number of qualitative studies were identified throughout the search process and future reviews could synthesise these findings to gain a deeper insight into the factors that influence uptake from the perspective of patients and practitioners.

\section{Conclusions}

This review found that, despite being the most widely used invitation method, letter invitations were less effective than telephone and face-to-face invitations (although one study revealed that this pattern may differ by patient gender and ethnicity). Nevertheless, there is evidence that letter invitations can be successfully enhanced using behavioural insights to improve uptake, which may be beneficial to services for whom telephone invitations are too costly. Our findings suggest that practices may need to consider additional targeted approaches to encourage groups who were found to have lower uptake rates of NHSHCs (namely younger cohorts, men, and those considered high risk according to lifestyle factors) to attend a NHSHC, however it was unfortunately not possible to find sufficient literature to identify which approaches may be best placed to reduced health inequalities. This review also finds that individual practice characteristics play a role in influencing uptake. As well as exploring how different demographic groups respond to invitation methods and interventions, further research is needed to understand the specific practice characteristics that impact NHSHC uptake, and whether the majority of such variation is due to the demographic characteristics of the patient list, or whether it is due to other factors such as availability of appointments.

With lifestyle factors now the biggest cause of death in the Western World it is imperative to reduce these lifestyle risk factors to achieve better health and wellbeing and reduce the associated health care costs.. It is clear further research is needed to help us identify effective ways of engaging people in preventative programmes such as the NHS HC. Understanding what interventions and invitation methods increase the uptake of NHSHCs, and identifying whether the efficacy of these interact with broader patient and contextual factors, will enable us to better support patients to reduce their risk. It is clear further research is needed to help us identify effective ways of engaging people in preventative programmes such as the NHS HC. 


\section{Supplementary information}

Supplementary information accompanies this paper at https://doi.org/10. 1186/s12889-019-7889-4.

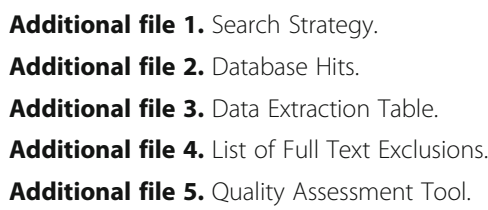

\section{Abbreviations}

BMI: Body mass index; CVD: Cardiovascular disease; DARE: Database of Abstracts of Reviews of Effects; GP: General practitioner; LG: Local government; NHSHC: National Health Service Health Check; PICOS: PatientIntervention-Comparator-Outcome-Study type; PRISMA: Preferred Reporting Items for Systematic reviews and Meta-Analyses

\section{Acknowledgements}

Nicola Pearce-Smith (re-ran searches 2018) and Dr. Belinda Hemmingway (quality assessment).

\section{Authors' contributions}

AKB conducted the original searches, selected manuscripts for inclusion, extracted data, and wrote the original report. LP assessed papers from the second round of searches, extracted data and updated the revised report. NG helped with interpretations of data and revisions to the manuscript. VB supervised the project. All authors read and approved the manuscript.

\section{Funding}

This work was originally conducted as a doctoral assignment by Amanda Bunten. Updated searches and further amendments were made as part of the usual business activities of PHEBI. Therefore, no funding bodies played a role in the design of the study, the collection, analysis or interpretation of the data, or the writing of the manuscript.

\section{Availability of data and materials}

Data sharing is not applicable to this article as no datasets were generated or analysed during the current study. All relevant materials for replicating this research (e.g., search strategies) are provided, and the reviewed studies are clearly referenced so that other researchers can access and assess the conclusions drawn in this paper. The data extraction table is provided in the additional files, which identifies the results that were drawn from various papers to inform the conclusions of this review.

Ethics approval and consent to participate

Not applicable (systematic review of previously published data).

\section{Consent for publication}

Not applicable (no individual information or data presented).

\section{Competing interests}

Amanda Bunten, Lucy Porter and Natalie Gold work for Public Health England Behavioural Insights (PHEBI). Amanda Bunten is second author of one of the studies included in the review.

\section{Author details}

${ }^{1}$ Public Health England, PHE Behavioural Insights Team (PHEBI), Research, Translation \& Innovation Division, 6th Floor, Wellington House, 133-155 Waterloo Road, London SE1 8UG, UK. ${ }^{2}$ University of Exeter, Exeter, UK. ${ }^{3}$ City University, London, UK.

Received: 24 June 2019 Accepted: 1 November 2019

Published online: 21 January 2020

\section{References}

1. World Health Organization. Cardiovascular Diseases 2017 [Available from: https://www.who.int/en/news-room/fact-sheets/detail/cardiovasculardiseases-(cvds).
2. Public Health England. Using the world leading NHS Health Check programme to prevent CVD 2018 [Available from: https:/www.gov.uk/government/ publications/using-the-nhs-health-check-programme-to-prevent-cvd/usingthe-world-leading-nhs-health-check-programme-to-prevent-cvd.

3. Department of Health. Putting prevention first vascular checks: risk assessment and management, impact assessment. London: Department of Health; 2008. [Available from: https://webarchive.nationalarchives.gov.uk/201 00408051934/http://www.dh.gov.uk/en/Publicationsandstatistics/Legislation/ Regulatoryimpactassessment/DH_091697

4. Public Health England. NHS Health Check Best practice guidance. London: Public Health England; 2017. [Available from: https://www.healthcheck.nhs. uk/commissioners-and-providers/national-guidance/

5. Alageel S, Gulliford MCJPm. Health checks and cardiovascular risk factor values over six years' follow-up: Matched cohort study using electronic health records in England. PLoS Med.2019;16(7):e1002863.

6. Usher-Smith JA, Mant J, Martin A, Harte E, MacLure C, Meads C, et al. NHS health check Programme rapid evidence synthesis. Cambridge: The Primary Care Unit, University of Cambridge; 2017.

7. NHS England. Programme Resources 2019 [Available from: https://www healthcheck.nhs.uk/commissioners_and_providers/delivery/invitation_letter_ and_results_card/.

8. Department of Health. Economic Modelling For Vascular Checks. London: Department of Health; 2008. [Available from: https://webarchive.nationalarchives. gov.uk/20130123194328/http://www.dh.gov.uk/en/Publicationsandstatistics/ Publications/PublicationsPolicyAndGuidance/DH_085869

9. Gidlow C, Ellis N, Randall J, Cowap L, Smith G, lqbal Z, et al. Method of invitation and geographical proximity as predictors of NHS health check uptake. J Public Health. 2014;37(2):195-201.

10. Dryden R, Williams B, McCowan C, Themessl-Huber M. What do we know about who does and does not attend general health checks? Findings from a narrative scoping review. BMC Public Health. 2012;12(1):723.

11. Cooper A, Dugdill L. Evidence of improved uptake of health checks: Rapid review 2014 [Available from: http://usir.salford.ac.uk/id/eprint/31864/.

12. Liberati A, Altman DG, Tetzlaff J, Mulrow C, Gøtzsche PC, loannidis JP, et al. The PRISMA statement for reporting systematic reviews and meta-analyses of studies that evaluate health care interventions: explanation and elaboration. PLoS Med. 2009;6(7):e1000100.

13. CASP. CASP Checklists 2019 [Available from: https:/casp-uk.net/casp-tools-checklists/.

14. Huang X, Lin J, Demner-Fushman D. Evaluation of PICO as a knowledge representation for clinical questions. AMIA annual symposium proceedings: American Medical Informatics Association; 2006. https://www.ncbi.nlm.nih. gov/pmc/articles/PMC1839740/\#_ffn_sectitle.

15. Cohen J. A coefficient of agreement for nominal scales. Educ Psychol Meas. 1960:20(1):37-46.

16. McHugh ML. Interrater reliability: the kappa statistic. Biochem Med. 2012; 22(3):276-82.

17. Popay J, Roberts H, Sowden A, Petticrew M, Arai L, Rodgers M, et al. Guidance on the conduct of narrative synthesis in systematic reviews. A product from the ESRC methods programme Version, vol. 1; 2006. p. b92.

18. Sterne JA, Egger M, Moher D. Addressing reporting biases. Cochrane handbook for systematic reviews of interventions: Cochrane book series; 2008. p. 297-333.

19. Attwood S, Morton K, Sutton S. Exploring equity in uptake of the NHS health check and a nested physical activity intervention trial. J Public Health (Oxf). 2016;38(3):560-8

20. Artac M, Dalton AR, Majeed A, Car J, Huckvale K, Millett C. Uptake of the NHS health check programme in an urban setting. Fam Pract. 2013;30(4):426-35.

21. Sallis A, Bunten A, Bonus A, James A, Chadborn T, Berry D. The effectiveness of an enhanced invitation letter on uptake of National Health Service Health Checks in primary care: a pragmatic quasi-randomised controlled trial. BMC Fam Pract. 2016;17:35

22. Coghill N, Garside L, Montgomery AA, Feder G, Horwood J. NHS health checks: a cross- sectional observational study on equity of uptake and outcomes. BMC Health Serv Res. 2018;18(1):238.

23. Cochrane T, Gidlow CJ, Kumar J, Mawby Y, lqbal Z, Chambers RM. Cross-sectional review of the response and treatment uptake from the NHS health checks programme in Stoke on Trent. J Public Health (Oxf). 2013;35(1):92-8.

24. Dalton AR, Bottle A, Okoro C, Majeed A, Millett C. Uptake of the NHS health checks programme in a deprived, culturally diverse setting: cross-sectional study. J Public Health (Oxf). 2011;33(3):422-9.

25. McDermott L, Cornelius V, Wright AJ, Burgess C, Forster AS, Ashworth M, et al. Enhanced invitations using the question-behavior effect and financial 
incentives to promote health check uptake in primary care. Ann Behav Med. 2018;52(7):594-605.

26. Cook EJ, Sharp C, Randhawa G, Guppy A, Gangotra R, Cox J. Who uses NHS health checks? Investigating the impact of ethnicity and gender and method of invitation on uptake of NHS health checks. Int J Equity Health. 2016;15(1):13.

27. Sallis A, Sherlock J, Bonus A, Saei A, Gold N, Vlaev I, et al. Pre-notification and reminder SMS text messages with behaviourally informed invitation letters to improve uptake of NHS health checks: a factorial randomised controlled trial. BMC Public Health. 2019;19(1):1162.

28. Sallis A, Gold N, Agbebiyi A, James RJE, Berry D, Bonus A, Vlaev I, Chadborn T. Increasing uptake of National Health Service Health Checks in primary care: a pragmatic randomized controlled trial of enhanced invitation letters in Northamptonshire, England. Journal ofPublic Health. 2019. https://doi. org/10.1093/pubmed/fdz134.

29. Conner M, Godin G, Norman P, Sheeran P. Using the question-behavior effect to promote disease prevention behaviors: two randomized controlled trials. Health Psychol. 2011;30(3):300.

30. Gold N, Durlik C, Sanders JG, Thompson K, Chadborn T. Applying behavioural science to increase uptake of the NHS Health Check: a randomised controlled trial of gain-and loss-framed messaging in the national patient information leaflet. BMC public health. 2019;19(1):1519.

31. Gidlow CJ, Ellis NJ, Riley V, Chadborn T, Bunten A, Iqbal Z, et al. Randomised controlled trial comparing uptake of NHS health check in response to standard letters, risk-personalised letters and telephone invitations. BMC Public Health. 2019;19(1):224.

32. Everett T, Bryant A, Griffin MF, Martin-Hirsch PP, Forbes CA, Jepson RG. Interventions targeted at women to encourage the uptake of cervical screening. The Cochrane Library; 2011.

33. ECM H, Martin A, Saunders CL, Meads C, Walter FM, et al. Reasons why people do not attend NHS Health Checks: a systematic review and qualitative synthesis. Br J Gen Pract. 2018;68(666):e28-35.

34. Brangan E, Stone TJ, Chappell A, Harrison V, JJHE H. Patient experiences of telephone outreach to enhance uptake of NHS Health Checks in more deprived communities and minority ethnic groups: A qualitative interview study. 2019;22(3):364-72.

35. Palmer CK, Thomas MC, McGregor LM, von Wagner C, RJBph R. Understanding low colorectal cancer screening uptake in South Asian faith communities in England-a qualitative study. BMC Public Health. 2015;15(1):998.

36. Hasvold PE, Wootton R. Use of telephone and SMS reminders to improve attendance at hospital appointments: a systematic review. J Telemed Telecare. 2011;17(7):358-64.

37. Waller D, Agass M, Mant D, Coulter A, Fuller A, Jones L. Health checks in general practice: another example of inverse care? Bmj. 1990;300(6732): 1115-8.

38. Expert Scientific and Clinical Advisory Panel. Emerging evidence on the NHS Health Check: findings and recommendations 2017 [Available from: http:// www.healthcheck.nhs.uk/commissioners-and-providers/evidence/.

39. Burgess C, Wright AJ, Forster AS, Dodhia H, Miller J, Fuller F, et al. Influences on individuals' decisions to take up the offer of a health check: a qualitative study. Health Expect. 2015;18(6):2437-48.

40. Jenkinson CE, Asprey A, Clark CE, Richards SH. Patients' willingness to attend the NHS cardiovascular health checks in primary care: a qualitative interview study. BMC Fam Pract. 2015;16(1):33.

41. Roberts D, de Souza V. A venue-based analysis of the reach of a targeted outreach service to deliver opportunistic community NHS health checks to 'hard-to-reach'groups. Public Health. 2016;137:176-81.

42. PHE. Improving people's health: applying behavioural and social sciences Gov.uk 2018 [Available from: https://www.gov.uk/government/publications/ improving-peoples-health-applying-behavioural-and-social-sciences.

\section{Publisher's Note}

Springer Nature remains neutral with regard to jurisdictional claims in published maps and institutional affiliations.

Ready to submit your research? Choose BMC and benefit from:

- fast, convenient online submission

- thorough peer review by experienced researchers in your field

- rapid publication on acceptance

- support for research data, including large and complex data types

- gold Open Access which fosters wider collaboration and increased citations

- maximum visibility for your research: over $100 \mathrm{M}$ website views per year

At BMC, research is always in progress.

Learn more biomedcentral.com/submissions 